\title{
Un rituel du monastère tibétain de Shéchen
}

Des textes à la pratique en terre d'exil

\section{Mireille Helffer}

\section{(2) OpenEdition}

Journals

Édition électronique

URL : http://journals.openedition.org/assr/23137

DOI : $10.4000 /$ assr.23137

ISSN : 1777-5825

Éditeur

Éditions de l'EHESS

\section{Édition imprimée}

Date de publication : 1 avril 2011

Pagination : 121-137

ISBN : 978-2-7132-2302-0

ISSN : 0335-5985

\section{Référence électronique}

Mireille Helffer, «Un rituel du monastère tibétain de Shéchen », Archives de sciences sociales des religions [En ligne], 154 | Avril-juin 2011, mis en ligne le 01 août 2011, consulté le 01 mai 2019. URL http://journals.openedition.org/assr/23137 ; DOI : 10.4000/assr.23137 


\section{Mireille Helffer}

\section{Un rituel du monastère tibétain de Shéchen Des textes à la pratique en terre d'exil}

Après la disparition du Bouddha historique Shâkyamuni, son enseignement s'est répandu en Asie selon plusieurs "voies» ou véhicules (tib. theg pa: skt. $y \bar{a} n a){ }^{1}$; on distingue : "le petit véhicule » (binayāna), "le grand véhicule » (mahayāna), "le véhicule adamantin » (vajrayāna, tib. rdo rje theg pa), dit aussi "véhicule des mantras" (mantrayāna, tib. gsang sngags gyi theg pa). Pour ce qui concerne les régions de culture tibétaine, la transmission s'est effectuée par la voie du mahayâna en différentes étapes.

On distingue en effet une " première diffusion » (snga 'dar) au cours de laquelle a été introduit un système d'écriture inspiré de l'Inde. C'est aussi l'époque (VIII siècle) où, grâce à l'intervention d'un thaumaturge indien, Padmasambhava dit aussi Guru Rinpoche ${ }^{2}$, a été construit le monastère de Samyé (tib. Bsam yas) et où a été entreprise la traduction des textes canoniques du sanskrit en tibétain par des couples d'érudits-traducteurs (lo tsa $b a$ ) formés d'un indien et d'un tibétain ${ }^{3}$.

À partir des $\mathrm{X}^{\mathrm{e}}-\mathrm{XI}^{\mathrm{e}}$ siècles, on a assisté à une seconde diffusion considérée comme phyi 'dar au cours de laquelle se sont différenciées les principales écoles du bouddhisme tibétain : "les anciens " ou nyingmapa (tib. rnying ma pa), " ceux de la tradition orale » ou kagyüpa (tib. bka’ brgyud $p a$ ), « ceux du monastère de Sa skya » ou Sakyapa (tib. Sa skya pa), «les vertueux » ou gelukpa (tib. dge

1. Précisions concernant les termes tibétains dont il est fait usage dans cet article : pour les termes techniques: la translittération en italiques - selon le système Wylie communément adopté dans le monde occidental - et l'indication éventuelle du terme sanskrit (skt.) qu'ils traduisent ont été adoptées. La transcription est plus ou moins phonétique pour les termes les plus courants dont certains figurent déjà dans les dictionnaires français. Pour les noms propres : la translittération (sans italique) a été privilégiée, et lorsqu'elles existent, nous avons adopté les transcriptions communément utilisées dans les publications en français ou anglais auxquelles cet article se réfère voire celles qui ont été retenues sur les sites internet des monastères.

2. Rinpoche signifie précieux et on peut traduire le titre Guru Rinpoche par Maître très précieux.

3. Pour mener à bien ce travail, a été élaboré le dictionnaire sanskrit-tibétain connu sous le nom de Mahãvyutpatti, comportant plus de dix mille entrées, classées par sujets et non par ordre alphabétique. 
lugs pa). Cette période a vu la constitution du volumineux canon bouddhiste, patrimoine commun des différentes écoles, réparti entre Kanjur ( $b k a$ ' 'gyur) et Tanjur (bstan 'gyur) ; ces deux collections qui regroupent plus de quatre mille cinq cents ouvrages, contenant, d'une part, les enseignements supposés du Bouddha historique et, d'autre part, des commentaires de tous ordres, ont fait l'objet d'un classement par le savant gelukpa Bu ston rin chen sgrub (1290-1364).

Par ailleurs, des "textes-trésors" (gter-ma), supposés cachés au temps de Padmasambhava, ont progressivement été mis au jour par des " découvreurs de trésors " (gter ston), principalement chez les nyingmapa et les kagyüpa. Ils ont été à la source de nombreux cycles rituels.

Enfin le développement de grands monastères-universités abritant parfois des milliers d'étudiants a offert un cadre propice aux études tout en donnant naissance à un système politico-religieux dominé par les gelukpa, au sein desquels se détachaient le Dalaï Lama et le Panchen Lama ${ }^{4}$.

Durant la seconde moitié du XXe siècle, et plus particulièrement après 1959 , l'invasion chinoise et la destruction massive des monastères ont entraîné une situation politique nouvelle dans laquelle les populations d'origine tibétaine se sont trouvées réparties entre la «Région Autonome du Tibet » (Xizang) proclamée en 1965 et les provinces chinoises de Gansu, Sichuan, Yunnan et Qinghai. Les principaux maîtres religieux des différents ordres, suivis souvent par des groupes importants de fidèles, ont pris le chemin de l'exil vers les régions frontalières de l'Inde, du Népal et du Bhoutan, tandis que le Dalaï Lama s'installait à Dharamsala devenue en quelque sorte la capitale du bouddhisme tibétain en exil.

Du côté de la tradition de l'école nyingmapa sur laquelle je m’appuirai plus particulièrement pour réfléchir à la relation entre monachisme et érudition, il y a lieu de souligner la coexistence de moines ordonnés (dge slong) et de religieux non célibataires (sngags pa). Les plus grands maîtres nyingmapa qui ont pris le chemin de l'exil appartiennent à l'une ou l'autre catégorie et l'on parle facilement de leur famille. C'était le cas pour Dudjom Rinpoché (1903-1987) aussi bien que pour Dilgo Khyentsé Rinpoché (1910-1991) (Ricard, 1996) qui ont successivement représenté les nyingmapa auprès du Dalaï Lama. Mais Padnor Rinpoché (1932-2009), était un moine, à la tête de l'école nyingmapa. C'est aussi à un

4. Lama signifie maître et dalaï océan. Dalaï Lama est un titre qui fut donné par les Mongols au chef religieux à partir de 1577. Panchen Lama signifie littéralement Maître grand érudit ; ce titre a été donné aux incarnations successives des abbés du monastère de Tashilhunpo. Les titres ou les termes d'adresse sont nombreux dans le bouddhisme: bla ma: skt. guru [bla na med $p a=$ sans supérieur], $c f$. Dalai Lama/Panchen Lama, rin po che : "Précieux ", appelation traduisant le respect [équivalent pour nous à Monseigneur], $c f$. Khyentse Rinpoché/Rabjam Rinpoché, lotsava (abrégé lo) : traducteur, cf. Lo chen Dharmashri/Marpa lotsaba, pandit: érudit/lettré, cf. Sakya Pandita/Pan chen Lama, gter bdag/gter ston : découvreur de " trésors ", $c f$. Gter bdag gling pa, mkhas sgrub : désigne un érudit (mkhas pa) dont les réalisations spirituelles sont accomplies (sgrub pa). 
moine, le respecté Trülshik Rinpoché (né en 1915), qu'a été confiée la charge de trouver la réincarnation de Dilgo Khyentsé Rinpoché.

Paradoxalement, suite aux événements douloureux qui ont marqué la fin du $\mathrm{Xx}^{\mathrm{e}}$ siècle et aboutissent aujourd'hui à une véritable colonisation du Tibet par la Chine, on a pu assister à un intense développement de la tibétologie dans le monde occidental, essentiellement dû à quatre facteurs : la présence et l'activité en exil de maîtres tibétains éminents, l'édition en Inde de nombreux textes tibétains (manuscrits et/ou xylographies) apportés par les exilés ${ }^{5}$, bientôt suivie par des éditions bon marché en Chine, la multiplication des traductions en anglais, en allemand et en français... et enfin la prolifération de centres bouddhiques en Occident.

Cette situation inédite fait qu'aujourd'hui certains vont jusqu'à parler d'une « diffusion ultérieure » (yang 'dar) du bouddhisme.

\section{Le monastère de Shéchen et son implantation au Népal}

Le monastère mère de Shéchen est le dernier en date des grands monastères de la tradition nyingmapa ${ }^{6}$. Fondé au Kham (actuelle province chinoise de Sichuan) en 1735, par Zhe chen Rab 'byams II 'Gyur med kun bzang rnam rgyal, il se réclame de la tradition du monastère de Mindroling, fondé au Tibet central par deux érudits : Gter bdag gling pa (1646-1714) et son frère Smin gling Lo chen Dharmashri (1654-1717). Sur les traditions du monastère de Mindroling, on dispose aujourd'hui d'un ouvrage facilement accessible, publié par les presses chinoises, en 1992, sous le titre : O rgyan Smin grol gling gi dkar chag ${ }^{7}$.

Le monastère mère de Shéchen a été, comme beaucoup d'autres, presque complètement détruit par l'occupant chinois, mais sa reconstruction progressive s'est effectuée, à partir de 1985, grâce au soutien matériel de Dilgo Khyentse Rinpoché qui y avait effectué une partie de ses études.

L'activité inlassable de ce maître prestigieux, un des plus respectés de l'école nyingmapa, qui avait trouvé refuge au Bhoutan avec sa famille dès 1959, s'est exercée dans une multitude de domaines, qu'il s'agisse de la publication

5. Grâce à l'activité intense du regretté Gene Smith (1936-2010), qui représentait la Library of Congress à New Delhi, des milliers de textes ont été recueillis et classés dans le Tibetan Buddhist Resource Center (TBRC).

6. Les six grands monastères de la tradition nyingmapa sont Kathog (Kah thog), fondé au XII ${ }^{\mathrm{e}}$ siècle puis restauré au XVII ${ }^{\mathrm{e}}$ siècle ; "Rocher adamantin " Dordjédrag (Rdo rje brag), fondé en 1632 ; Pëlyul (Dpal yul), fondé en 1665 ; Mindroling (O rgyan Smin grol gling), fondé en 1676 ; Dzogchen (Dzogs chen dgon pa), fondé en 1685 et qui était le plus important en nombre avant 1959 avec environ mille moines et, finalement, Shéchen (Zhe chen bstan gnyis dar rgyas gling), fondé en 1735. Cf. Cornu (2001 : 614), voir la carte "Les grands monastères du Tibet ".

7. Il s'agit d'une somme de renseignements très précis concernant le monastère, sa construction, ses activités et son calendrier liturgique. 
de textes fondamentaux de la tradition bouddhique ou de nombreux ouvrages nyingmapa, de la transmission des enseignements dont il était dépositaire grâce à son immense culture, de la construction en exil d'un nouveau monastère de Shéchen (Ricard, 1996).

En ce qui concerne les éditions de textes fondamentaux appartenant aussi bien à la catégorie canonique des tantras, qu'à celle des "trésors cachés » (gter $m a)$, et enfin à la tradition orale des $b k a^{\prime} m a$, c'est sous ses auspices qu'ont été notamment publiés de nombreuses collections. On peut citer :

- «les 100000 tantras anciens »(Rnying ma rgyud 'bum) rassemblés par les fondateurs de Mindroling et revus plus tard par'Jigs med gling pa (1730-1798), publiés à Delhi en 1970 ;

- «le trésor des enseignements redécouverts » (Rin chen gter mdzod), en soixantetrois volumes, compilé par un maître éminent du mouvement non sectaire ris-med ${ }^{8}$ : Kong sprul (1813-1899);

- l'ensemble des "enseignements transmis oralement de maître à disciple " regroupés sous le titre de $B k a^{\prime}$ ma par O rgyan Gter bdag gling pa et Smin gling Lo chen Dharmashri (transmission de Bdud 'joms Rinpoché (1904-1987) à Khrul zhig Rinpoché ;

- le Gnam chos thugs kyi gter kha snyan brgyud zab mo’i skor (titre abrégé : Gnam chos) de Mi 'gyur rdo rje (1645-1667), publié en treize volumes au Bhoutan en 1983 ; l'édition en a été possible grâce au jeu d'imprimés de l'édition xylographique de Rmug sangs, conservés à Paris dans le fonds Migot de l’École Française d'Extrême-Orient.

Non content de promouvoir l'édition de ces textes essentiels, Dilgo Khyentsé Rinpoché a également poursuivi sans relâche la transmission orale des enseignements dont il était dépositaire en octroyant non seulement les «transmissions de pouvoir» (dbang: skt. abhisheka), mais aussi en procédant aux interminables lectures orales d'autorisation (lung: skt. agama) préalables à l'étude proprement dite des textes et éventuellement complétées par des instructions (khrid), d'abord au Bhoutan, puis en Inde et au Népal, avant d'aborder le monde occidental.

La construction du Shéchen népalais, à proximité du grand stupa de Bodnath, initiée au début des années quatre-vingt, a impliqué des activités multiples alliant construction, conservation des textes propres à cette tradition monastique, organisation des enseignements, accueil et entretien des jeunes « novices » qui affluaient aux portes du nouveau monastère, organisation des parrainages et de leur financement. Elle s'est poursuivie grâce au soutien matériel de la famille royale du Bhoutan dont Khyentse Rinpoché était le chapelain. C'est ainsi que de nombreux

8. Ce mouvement s'est organisé à la fin du XIX ${ }^{\mathrm{e}}$ siècle et a réuni des maîtres de toutes les traditions du bouddhisme tibétain. 
artistes bhoutanais ont contribué à la décoration de l'édifice, si bien que localement le monastère était alors souvent désigné par le titre : "monastère bhoutanais » ou monastère de Khyentsé Rinpoché.

La préservation des documents écrits relatifs à la tradition du monastère de Shéchen a bénéficié pour sa part de l'action efficace du « Nepal German Manuscript Preservation Project » financé par l'Allemagne, grâce auquel tous les documents manuscrits et imprimés disponibles ont été systématiquement photographiés et indexés.

Simultanément se manifestait le souci de la mise en place du calendrier liturgique, conformément au modèle de Mindroling, même si certaines dates étaient modifiées en raison des conditions climatiques. Ce fut notamment le cas pour la grande fête du « dixième jour du mois » (Tshes bcu) célébrée en l'honneur de Padmasambhava; elle était originellement célébrée le dixième jour du cinquième mois du calendrier tibétain et fut transférée au dixième jour du deuxième mois.

Enfin, il fallait d'urgence instituer et organiser sur place un véritable cursus des études, assurant à la fois le niveau élémentaire pour l'enseignement des jeunes recrues, la formation de maîtres qualifiés, l'organisation des études supérieures avec des cycles courts pour ceux dont les capacités intellectuelles ou les dispositions personnelles ne leur permettaient pas d'envisager les cycles longs d'un " collège philosophique " (bshad grwa) requérant neuf années d'études sanctionnées par des examens. Le programme du bshad grwa est extrêmement étendu et exigeant, il comprend l'étude de la philosophie, de la dialectique, des arts... Cependant, tous les moines ne font pas des études et ne sont pas des érudits.

\section{Mon premier contact avec le monastère de Shéchen}

Les observations qui suivent résultent de mes visites personnelles au monastère de Shéchen en exil et furent précédées de plusieurs rencontres avec Dilgo Khyentsé Rinpoché - en Inde, au Népal ou en France. Mais un pas décisif a été franchi quand je suis arrivée au Shéchen népalais, en 1989, invitée par le petitfils de Dilgo Khyentse Rinpoché, l'actuel Rabjam Rinpoché (né en 1966) septième de la lignée des Rab 'byams - que j'avais rencontré lors d'un grand rassemblement autour de la lecture solennelle (lung) des œuvres de Mi pham (1846-1912) tenu, en 1987, au monastère de Dpal Yul dans la province du Karnataka au sud de l'Inde.

À mon arrivée, le monastère abritait environ deux cents « moines ", dont la plupart étaient de jeunes novices confiés au monastère par leurs parents (Tibétains réfugiés, Népalais, Bhoutanais...) Mon enquête portait alors sur les pratiques musicales du monastère et devait se poursuivre jusqu'en 2001 (Helffer, 1995 ; 2004a et b ; 2005 ; notice du CD Sounds of Tibet). Cependant, je constatai bien vite qu'il me fallait d'abord acquérir la connaissance (au moins superficielle) des 
rituels auxquels j'étais autorisée à assister et qui appartenaient pour la plupart à la catégorie des sgrub chen et sgrub mchod, à commencer par l'important rituel qui marquait la fin de l'année le dgu gtor/gtor zlog et se déroulait du vingt et unième au vingt-neuvième jour du douzième mois du calendrier tibétain (Helffer, 2004).

S’y ajoutèrent, au cours du premier mois du calendrier tibétain, la célébration de «la grande assemblée de toutes les divinités " (Tshogs chen 'dus pa), puis celle de "Rdo rje sems dpa' [selon la tradition] de Mindroling " (titre abrégé : Smin gling rdor sems), bientôt suivie par la grande fête du "dixième jour » [du mois], le Tshes bcu. Certaines de ces célébrations étaient suivies par les spectaculaires "offrandes ignées" (sbyin sreg, skt. homa) dans la cour du monastère et/ou l'exécution de ce qu'on peut considérer comme un ballet rituel ('chams).

Pour tenter de suivre ces différents rituels, il m'a fallu collecter des photocopies des textes et manuels divers utilisés, opération largement facilitée par la présence au monastère du moine français Matthieu Ricard qui faisait fonction de secrétaire de Dilgo Khyentsé Rinpoché. Rappelons ici quelques-unes des affirmations du tibétologue et historien des religions Per Kvaerne à propos d'un rituel qu'il avait observé en Inde : « les rituels dépendent toujours des textes (...) même les textes ne sont que provisoires (...) ils impliquent la récitation des mantras sans lesquels le rituel resterait inefficace (...) un rituel se compose d'un certain nombre d'éléments de base qui sont choisis, répétés, combinés ou élaborés selon le but et la nature du rituel à accomplir. » (Blondeau, Schipper, vol. I : 147-148).

Ayant obtenu la permission d'assister aux rituels effectués à cette époque de l'année, je pris l'habitude de m'installer discrètement à une place située derrière le dernier rang des moines les plus jeunes et j'appris progressivement à suivre les textes des rituels, textes dont je me suis rapidement aperçue qu'ils étaient dus pour la plupart aux deux fondateurs du monastère de Mindroling. Ces rituels étaient fort développés puisque, pendant sept jours consécutifs, à raison de plusieurs heures par jour, ils mobilisaient toute la communauté et que, pendant la nuit, il y avait récitation permanente de mantras. À la réflexion, il semble que ma situation n'était alors pas très différente de celle des plus jeunes participants qui ne reçoivent aucune explication sur la signification des textes qu'ils sont supposés réciter par cœur ou déchiffrer. Aujourd'hui, on constate la présence physique de livres pendant l'office et les rituels alors qu'autrefois il fallait tout savoir par cœur.

C'est au cours des rituels que j'appris à distinguer les positions hiérarchiques et la répartition des responsabilités au sein de la communauté monastique. Il y avait la place éminente des "réincarnés " (sprul sku) dont les trônes plus ou moins élevés selon le rang reconnu à leur occupant dominaient toute l'assistance, le rôle essentiel qui revenait à l'officiant principal, maître du rituel tantrique ( $r d o$ rje slob dpon skt. vajracârya), préférentiellement choisi parmi les "réincarnés ", le rôle non moins essentiel du responsable de la partie musicale, « celui qui donne 
le départ » (dbu mdzad) sur lequel repose l'enchaînement des différentes parties, les fonctions dévolues aux professeurs (mkhan po), au maitre de la discipline (dge bskos), au sacristain (mchod dpon), les places respectives occupées par les moines ordonnés (dge slong), ceux qui ont pris pour la vie deux cent cinquantetrois vœux de la règle monastique, rangés par ordre de séniorité, ainsi que par les novices (dge tshul), ceux qui ont pris seulement les trente-six vœux et qui, pour la plupart, sont encore des enfants ou des adolescents ${ }^{9}$.

La possession des textes, si indispensable soit-elle pour mettre en place la célébration des rituels, ne saurait suffire, et Dilgo Khyentse Rinpoché, animé par le souci de rétablir aussi fidèlement que possible la liturgie du monastère mère, fit appel à d'anciens religieux venus du Tibet, capables de transmettre aux jeunes générations leur compétence et leur savoir-faire.

C'est ainsi que, pour ce qui concerne les traditions musicales, j'ai vu se succéder plusieurs maîtres de chant $(d b u$ mdzad) dont j'ai recueilli les enregistrements pour archivage. Il y eut tout d'abord « le barbu » 'Gyur med 'jigs med, suivi par Nor bzang qui appartenait à une génération plus jeune ${ }^{10}$.

Le rétablissement progressif des danses rituelles 'chams, qui font partie de la célébration des rituels les plus importants, mobilisa des compétences multiples qui étaient plutôt de l'ordre des savoir-faire : fabrication sur place de plus d'une

9. Quelques termes du vocabulaire tibétain relatif à l'état religieux méritent d'être précisés : Grwa pa: terme général désignant les membres de la communauté monastique, ceux qui portent l'habit de moine. Rab tu 'byung ba (abrégé : rab 'byung), étape d'entrée dans la communauté religieuse, marquée par la coupe d'une mèche de cheveux par le maître qui accueille. Il existe différents états des membres de la communauté monastique : -Dge 'dun sde gnyis dont rab 'byung dge 'dun gyi sde: les moines ordonnés (vêtus selon les règles énoncées dans les écrits canoniques) et gos dkar lcang lo can gyi sde : ceux qui sont vêtus de blanc et portent les cheveux longs (= ci-dessous sngags pa). Les ermites : rnal 'byor pa/skt. Yogin; les religieux n'ayant pas pris les vœux de moine, souvent qualifiés de «tantristes " : sngags pa; les novices ayant pris les trente-trois vœux : dge tshul : skt. shramanera ; les moines ayant reçu la pleine ordination selon les règles du vinaya, c'est-à-dire ayant pris les deux cent cinquante-trois vœux : dge slong : skt. bhiksu (NB : un moine a la possibilité de prendre ses vœux de façon temporaire ou définitive); les "réincarnés " : sprul sku et yang srid, les dge bshes : ayant passé leur doctorat en sciences religieuses. Les différentes charges sont généralement exercées de façon temporaire : l'abbé [enseignement] : mkhan po, le maître du rituel tantrique, apte à donner initiations et enseignements : rdo rje slob dpon/skt. vajrâcaryâa [peut être un sngags pa ou un moine ordonné], "celui qui prend la tête " [de la récitation] / le maître de chant : $d b u$ mdzad, celui qui est chargé de la discipline : dge bskos, le responsable des "offrandes" [sacristain] : mchod dpon et l'économe : gnyer $p a$, etc. Notons que dans le néobouddhisme, les différents titres His Holiness et Khyab rje sont fréquemment adoptés pour les dignitaires de haut rang.

10. C'est avec ce dernier que, à l'instigation de Rabjam Rinpoché, j’ai procédé, en 1995 , à l'enregistrement complet des deux volumineux manuels de chant dbyangs yig correspondant aux " protecteurs de la religion " (chos skyong) et à la "divinité d'élection » (yi dam) du monastère: Gshin rje gshed. À partir de 1997, les maîtres de chant en poste (renouvelables tous les trois ans) avaient été formés en exil à Bodnath et, à plusieurs reprises, ils me demandèrent de leur faire parvenir des copies des enregistrements réalisés auprès des deux maîtres précédents. C'est d'ailleurs sous la direction de l'un d'entre eux que furent réalisés les enregistrements figurant sur le CD Sounds of Tibet. 
centaine de masques et costumes, copie du livret décrivant les danses ('cham yig), invitation à Bodnath d'un ancien maître de danse expérimenté ('cham dpon) du nom de Drudi (Ricard 1999 : 50-51). Celui-ci devait rester plusieurs années au monastère et sut former, parmi les jeunes moines, toute une génération d'excellents danseurs ${ }^{11}$. Grâce à sa présence, furent progressivement rétablies des danses oubliées comme le rol 'cham (Ricard, 1999).

\section{L'héritage de Dilgo Khyentsé Rinpoché}

À la disparition de Dilgo Khyentsé Rinpoché, en 1991, son petit-fils et héritier spirituel, Rabjam Rinpoché, abbé du monastère, reprit le flambeau, tout en poursuivant la recherche de la "réincarnation " (yang srid) de Khyentsé Rinpoché, identifiée en 1993 et intronisée en 1997.

Les publications en tibétain se poursuivirent à grande échelle, mais aussi les traductions en français et en anglais par le biais des éditions Padmakara établies en Dordogne. Une édition en tibétain des Euvres complètes (Gsung 'bum) de Dilgo Khyentsé Rinpoché fut préparée sur place à Bodnath grâce à un équipement informatique performant auquel furent initiés plusieurs jeunes moines. En 1997, la collection complète des notations musicales (dbyangs yig) fit l'objet d'une publication très soignée à Delhi regroupant six recueils dont la composition est due à un "maitre de chant» qui vécut au début du XIX ${ }^{\mathrm{e}}$ siècle ${ }^{12}$. Il s'agit de six manuels correspondant à quelques-uns des cycles rituels les plus importants de la tradition nyingmapa et classés par ordre chronologique de leur élaboration : - le Bka' brgyad bde gshegs dus pa'i dbyangs yig (pp. 1-200), basé sur le gter ma découvert par Nyang ral nyi ma 'od zer (1136-1204) et désigné par le titre poétique "Son du dragon qui annonce la saison des pluies » (char ldan dus kyi 'brug sgra);

- le Bla ma'i gsang 'dus pa'i dbyangs yig (pp. 203-264), basé sur le texte-trésor (gter ma) découvert par Guru Chos dbang (1212-1270), et désigné par le titre "Grand tambour des dieux " (srid zhi kun khyab lha yi rnga chen) ${ }^{13}$;

- le Chos skyong rnams kyi dbyangs yig (pp. 267-425), basé sur une collection de textes relatifs aux divinités protectrices de la religion (chos skyong) et à leurs acolytes (rjes 'brang). Ces derniers textes sont dus aux deux fondateurs du monastère de Mindroling. Exceptionnellement, la date de composition de cet ouvrage, destiné à "réjouir les protecteurs de la religion » (bstan srung dgyes par byed $p a)$ et utilisé dans de nombreux rituels, figure dans le colophon et correspond à l'année 1822 de l'ère commune ;

11. J'ai pu apprécier leurs progrès au cours des années notamment lors des spectacles exportés à Turin, au Théâtre du Soleil, etc.

12. Sous-titre anglais : "A collection of musical notations for the rituals performed in the Nyingma Monastery of Shechen Tenyi Dargyeling ", 642 p.

13. Ce manuel est utilisé pour la célébration de la fête de Padmasambhava, le dixième jour (tshes $b c u)$ du deuxième mois du calendrier tibétain. 
- le Guru drag po’i dbyangs yig (pp. 439-480), correspondant à un rituel en l'honneur de la forme de Padmasambhava dite "Le maître farouche " (Guru drag po) et intitulé "Tambour de l'été » (dus kyi dbyar rnga). Le texte de référence est dû, comme le précédent, à Gter bdag gling pa ;

- le Slob dpon Sangs rgyas gnyis pa'i gsol 'debs Le'u bdun ma dbyangs yig (pp. 485-508). Il s'agit en réalité de la notation musicale pour la fameuse prière en «sept chapitres » (Le'u bdun ma) découverte par Rig 'dzin rgod ldem (13371408) intitulée "Les sept mélodies étonnantes" (Ngo mtshar dbyangs bdun); - le Gshin rje dregs 'joms byed kyi dbyangs kyi yi ge (pp. 509-639), manuel destiné à accompagner le rituel issu du gter ma relatif à la forme de Yamantaka dite Gshin rje dregs 'joms, découvert par Gter bdag gling pa en 1667, est désigné par le titre «Rugissement du lion» (Seng ge nga ro).

Tous ces dbyangs yig constituent en fait des sortes de partitions musicales, aide-mémoire indispensables à la célébration puisqu'ils indiquent non seulement, les « syllabes sans signification " (tshig lhad) sur lesquelles s'appliquent les vocalisations (dbyangs) des chants les plus élaborés, mais comportent aussi quantité d'informations : signes neumatiques placés au-dessus de certaines syllabes pour indiquer les inflexions vocales, indications relatives aux interventions instrumentales comme la clochette dril bu et le tambour damaru actionnés par le " maître du rituel », les cymbales conductrices tenues par le " maître de chant », les grands tambours rnga et les différentes catégories de trompes (conques dung dkar, trompettes rkang gling, longues trompes dung chen); le jeu de tous ces instruments étant confié à des musiciens de service (Helffer, 1994).
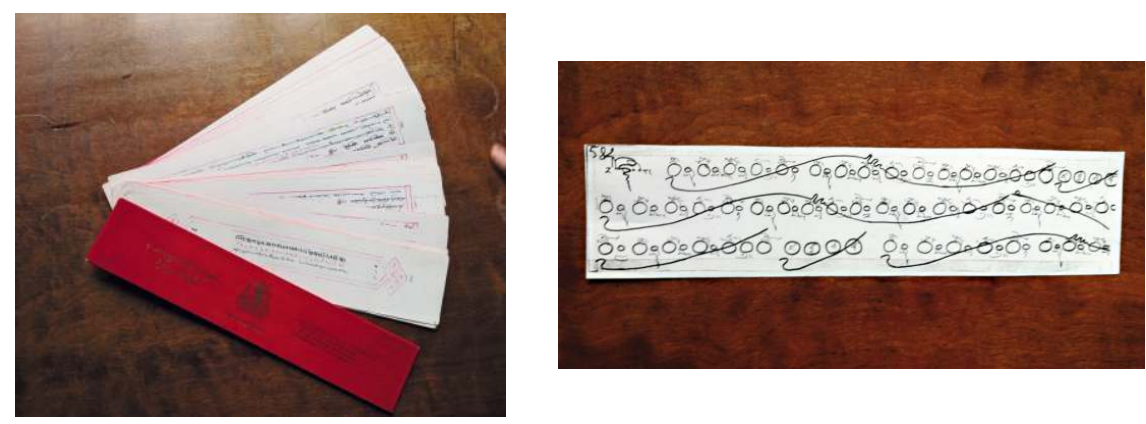

Notation pour le jeu simultané des tambours et des longues trompes recueillie au monastère de Shéchen, Népal (archives Mireille Helffer)

C'est en 2000 et 2001, dans le cadre du Shechen Project soutenu par la Commission européenne que furent publiés les exceptionnels « modes d'emploi » (laglen) pour les principaux rituels effectués au monastère de Shéchen, sous le titre Zhe chen lugs srol cho ga'i lag len dont le sous-titre anglais est "A set of unique manuals for the rituals in use at Shechen Tennyi Dargyeling monasteries 
in accordance to the Mindroling tradition of the Nyingma school of Tibetan Buddhism ». La préface, rédigée en anglais par M. Ricard, précise le contenu de chacun des volumes.

C'est également sous les auspices du monastère que furent publiés des usuels comme le Manuel de pratiques religieuses (Chos spyod), publié par le vingtquatrième Nyingma Students' Welfare Committee, Sarnath-Vanarasi U.P. (India, 1998). Il s'agit là d'un recueil de prières courantes ( $g$ sol 'debs), édité en format à l'occidentale. On peut y ajouter le Zhe chen bstan gnyis dar rgyas gling gi'don cha, (309 p.), compilation qui regroupe les textes relatifs à la célébration du rituel de "La réunion de la grande assemblée des divinités » (Tshogs chen 'dus $p a)$, selon les formes développée, moyenne et abrégée ainsi qu'enfin les textes concernant le protecteur Mgon po legs ldan.

Près du monastère à Bodnath a été érigée la série habituelle des huit stupas $(\text { mchod rten })^{14}$ et un grand moulin à prières, a été ouverte en 1995 une hôtellerie contiguë au monastère pour accueillir les visiteurs et les fidèles, la Rabsel Guest House, le Tesring Art School en 1997, un collège philosophique (bshad grwa), en 2001, puis un dispensaire. Dans la vallée de Katmandou, à Namo Buddha, fut créé un centre de retraite et à Bodhgaya (Inde) fut ouvert le Shechen Institute, en 1996. Il faut ajouter à cela l'aide à la reconstruction du Shechen tibétain et le développement d'un vaste programme humanitaire dans la même région grâce à la création de l'Association Karuna.

\section{La célébration du rituel de gtor zlog}

J'examinerai ici le cas du grand rituel de fin d'année dit gtor zlog ou $d g u$ gtor célébré du vingt et unième au vingt-neuvième jour du douzième mois du calendrier tibétain destiné à expulser les maux de l'année écoulée ${ }^{15}$. C'est un rituel auquel j'ai plusieurs fois assisté (Helffer, 2004a) et dont la célébration requiert la connaissance et la maîtrise d'un ensemble impressionnant de textes et manuels mis à ma disposition par les autorités monastiques dont on trouvera ci-dessous le descriptif :

14. Pour une illustration de ces monuments, voir Cornu (2001: 549-553).

15. Le vocabulaire tibétain relatif aux rituels mérite d'être précisé :

Chos: skt. Dharma désigne l'ensemble des enseignements dispensés par les bouddha et les maîtres spirituels et cho ga correspond à l'ordonnance des actes successifs d'une action (pas forcément rituelle).

rab gnas: consécration; sgrub thabs: skt. sâdhana: sgrub chen: rituel de " grande évocation " qui vise à la coercition d'une divinité au moyen d'opérations complexes de visualisation du mandala qui la représente ; sgrub mchod est une forme moins développée du sgrub chen; sbyin sreg: skt. Homa est un rituel du feu sacrificiel/offrandes ignées, gtor zlog : rituel pour détourner, chasser ou éviter les esprits nuisibles au moyen de "gâteaux rituels" (gtor ma). 
Les textes rituels : ces textes se présentent sous forme de feuillets mobiles écrits recto verso $(d p e c h a)$ que l'on peut organiser selon les enchaînements choisis par le maître du rituel. En somme, ces livres se font et se défont. Ils se lisent toujours dans le même ordre mais on peut en extraire certains feuillets pour n'en réciter que les morceaux requis par tel ou tel rituel. Dans ce cas particulier, tous les textes sont dus aux deux maîtres fondateurs du monastère de Mindroling. Ils concernent, d'une part, Gshin rje gshed - la divinité tutélaire (yi dam) du monastère - et ses acolytes et, d'autre part, les neuf divinités protectrices (chos skyong) de l'ordre nyingmapa.

Quant aux textes relatifs à Gshin rje gshed (skt. Yamantaka), sous sa forme terrible de dregs 'joms: le premier d'entre eux est extrait du gter mdzod Smin gling dregs 'joms et comprend, comme il se doit, le rappel de la lignée de transmission (brgyud 'debs) : 1-4, suivi du guide des actions rituelles (las byang) pour Gshin rje gshed et son principal acolyte Las gshin ; 5-29. Le second, intitulé 'jam dpal Gshin rje gshed dregs 'joms byed kyi drag po zor gyi man ngag rdo rje'i thog $m d a$ ' (titre abrégé : zor 'phrin), est composé d'une vingtaine de folios extraits des œuvres complètes de Dharmashri. Il a été recopié en caractères d'imprimerie ( $d b u c a n)$ pour en faciliter la lecture et l'usage liturgique. Comme l'indique le titre, il concerne les instructions (man ngag) relatives à l'emploi des projectiles (zor) dans le rituel basé sur le cycle de Gshin rje gshed dregs 'joms. La violence des actions à effectuer est soulignée par l'appellation " pointe de flèche adamantine" ( $r d$ o rje'i thog $m d a^{\prime}$ ). Il s'agit en fait du texte sur lequel, sans en identifier l'auteur, s'était appuyé Stein (1957) dans un article qui a fait date ${ }^{16}$.

On trouve également le texte relatif aux divinités protectrices (chos skyong) et à leurs auxiliaires (rjes 'brang). Il s'agit là d'une anthologie - titre abrégé : Smin gling dngos rol - qui regroupe des textes disparates consacrés, d'une part, aux neuf grands protecteurs de l'école nyingmapa et, d'autre part, à la douzaine de leurs auxiliaires (rjes 'brang): Bstan pa skyong ba'i dam can chen po rnams kyi phrin las dngos grub kyi rol mtsho : pp. 129-234, suivi de $r$ do rje chos skyong rnams kyi las byang dngos sgrub rol mtsho'i rjes 'brang gi 'phrin las bye brag rnams phyogs gcig tu bsdebs pa: pp. 235-253.

Comme l'a montré Kohn (2001) dans sa remarquable analyse du Mani Rimdu - une fête célébrée dans plusieurs monastères du pays sherpa au Népal -, ce texte, dont il fournit la traduction intégrale en anglais, a connu une large diffusion.

Les manuels de notations pour le chant (dbyangs yig) : Les deux plus volumineux d'entre eux sont destinés à accompagner les textes décrits ci-dessus ${ }^{17}$. Il s'agit

16. «Le linga des danses masquées lamaïques et la théorie des âmes » (Stein, 1957).

17. Ce sont ces deux recueils dont Rabjam Rinpoche m'a demandé d'assurer l'enregistrement avec le maître de chant Nor bzang, en 1995. 
du Chos skyong rnams kyi dbyangs yig bstan srung dgyes byed, le "manuel pour les protecteurs de la doctrine [bouddhique] intitulé ce qui fait la joie des gardiens de la loi religieuse » : pp. 267-425 du recueil publié en 1997 et dont la rédaction remonterait à 1822, ainsi que Gshin rje dregs 'joms byed kyi dbyangs yig seng ge'i nga ro, le "manuel pour Gshin rje gshed dregs 'joms [intitulé] le rugissement du lion» : pp. 509-630.

Le manuel relatif aux danses ('chams yig) : Des danses spectaculaires sont exécutées dans la cour du monastère et interviennent le vingt-huitième et le vingtneuvième jour de la fête, après la célébration du rituel proprement dit dont l'horaire a été avancé.

La copie manuscrite du 'chams yig, provenant du monastère mère est de lecture difficile et a été transférée sur ordinateur. Elle compte vingt-trois pages et a pour titre lo ming dus rim chen mo'i 'chams yig blo gsal dga' ba'i dpal: «manuel de danse pour la grande fête de la fin de l'année [intitulé] dont la splendeur donne la joie d'une compréhension claire ». On y trouve non seulement une description détaillée de la chorégraphie, mais aussi les indications relatives aux interventions instrumentales et le rappel de l'incipit des textes énoncés par le maître de chant, en référence aux données du manuel de chant.

Les danses, dont l'apprentissage intervient dans la formation des jeunes moines au cours du niveau moyen du cursus monastique, comportent : la « danse au tambour " rnga 'cham [par les " chapeaux noirs" (zhwa nag)], la « danse des maîtres des cimetières " (dur bdag), la "danse des gardiennes des portes » (sgo ma) [du mandala], la danse des deux oiseaux bya rog et 'ug pa, la danse des rigs bsdus [les divinités du mandala de Gshin rje gshed], il est procédé ensuite à la destruction-libération de l'effigie linga et enfin le zor 'chams ${ }^{18}$ au cours duquel sont détruits les grands gâteaux rituels (gtor $m a$ ) en forme d'effigies représentant Gshin rje gshed et son acolyte Las gshin (Ricard, 1999: 74-82 ; 84-85).

18. Zor : désigne différents matériaux et substances utilisés comme projectiles et lancés en direction d'un ennemi au cours d'un rituel. Zor 'phrin: titre abrégé du texte figurant dans les œuvres complètes de Dharmashri sous le titre 'jam dpal Gshin rje dregs 'joms kyi drag po zor gyi man ngag rdo rje'i thog mda' (cf. ci-dessus). Ce texte, dont la date de composition n'est pas connue, est utilisé à Shéchen pour la célébration du gtor zlog/dgu gtor [cf. Smin gling dkar chag: 214-220]. Dans la partie présentée comme zor bskul ba (fol. 20b), sont explicités les divers matériaux utilisés comme zor durant le zor 'cham, à savoir : gtor zor/khrag zor/mda' zor/rdo zor/thun zor/dug zor [pour une représentation de différents zor, Karmay, 1989, pl. 21]. Vient ensuite le zor 'phen pa (fol. 22b) qui correspond au lancer des différents zor en direction des deux grands gtor ma représentant Gshin rje gshed et Las gshin déposés dans une hutte en paille, avant leur mise à feu. Les cendres sont recueillies et déposées dans un trou prévu à cet effet. Puis la danse rta bro (fol. 22b) parachève le rituel. 


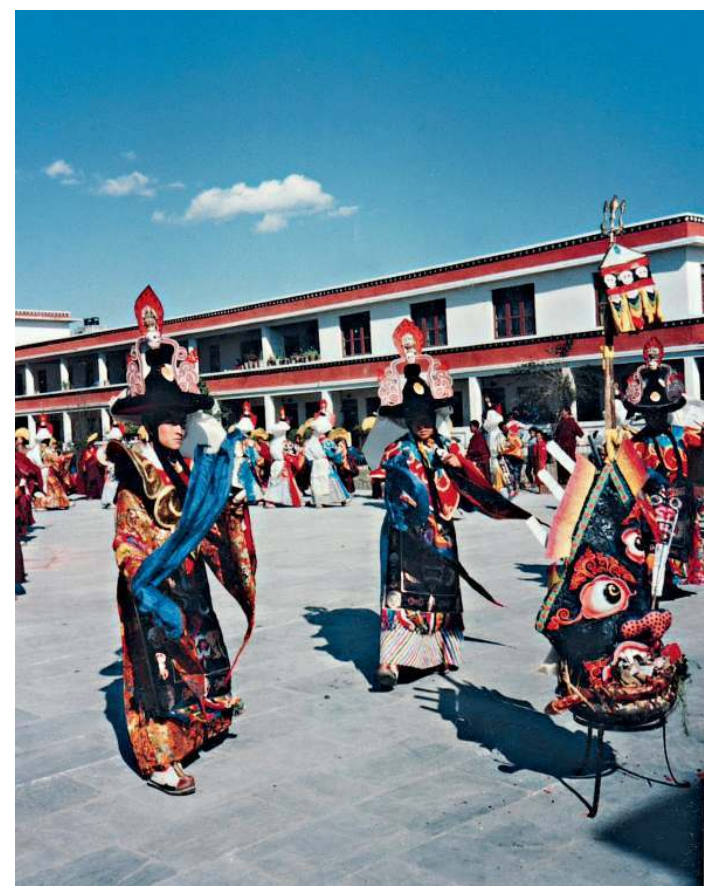

Danse rituelle. Monastère de Shéchen, Népal, Bodnath (Mireille Helffer 1994)

Le mode d'emploi (laglen): Les lag len constituent un rappel de la pratique coutumière d'un rituel et sont élaborés en tenant compte des données déjà présentes dans les manuels spécialisés. Ils s'adressent à des experts qui connaissent les différents textes à mettre en œuvre et, comme j’ai pu le constater à maintes reprises, ils sont principalement consultés par le maître de chant ( $d b u$ mdzad) et par le célébrant ( $r d o$ rje slob dpon) qui ont besoin de se rafraîchir la mémoire pour assurer correctement le déroulement d'une cérémonie célébrée une fois par an.

Comme l'exprime le titre, 'Jam dpal Gshin rje dregs pa 'joms byed kyi gtor zlog lag len, il s'agit en l'occurrence du "mode d'emploi » pour la célébration du rituel de gtor zlog centré sur le glorieux Gshin rje dregs pa 'joms byed - une forme farouche du bodhisattva Manjushri - intitulé "miroir de lapis-lazuli » (rab gsal baidurya me long), vol. 2 : 61-190.

\section{Conclusion}

Ce rappel, forcément limité et sommaire, des problèmes auxquels a fait face un monastère tibétain en situation d'exil, paraît illustrer assez clairement le rapport étroit qui, au cours de l'histoire mouvementée du bouddhisme tibétain, a associé les deux composantes monachisme et érudition. C'est, en effet, seulement 
dans un cadre monastique - et ceci est valable pour toutes les écoles du bouddhisme tibétain - que pouvaient être poursuivies les études permettant l'élaboration et la rédaction d'ouvrages fondamentaux dus à des érudits qui devaient disposer de bibliothèques fournies et maîtriser une littérature considérable. C'est dans les monastères qu'étaient transmises de maître à disciples les «autorisations » (dbang) et les interminables lectures à voix haute (lung) précédant l'accès aux textes. C'est au sein des monastères que s'exerçaient et s'exercent encore les " débats rituels " au cours desquels se développent les capacités intellectuelles des jeunes moines.

Le monastère devient alors le lieu de sauvegarde et de production de différents livres : ceux que l'on ne modifie pas (les livres de prières, par exemple) et les textes sur l'enseignement qui, de tout temps, ont proliféré. Cependant, au-delà des pratiques lettrées, il s'agira aussi pour les moines de pratiquer la méditation en faisant le vide de tout ce qui disperse l'esprit et en visualisant une divinité.

Quant aux nombreux rituels qui rythment le calendrier monastique, on a pu voir qu'ils sont tous basés sur des textes, composés dans une langue calquée sur la poésie sanskrite et très différente de la langue parlée. Dans le cas particulier de Shéchen, il s'agissait de textes dus principalement, sinon exclusivement, aux deux maîtres nyingmapa qui avaient fondé le monastère de Mindroling et qui étaient contemporains du grand cinquième Dalaï Lama (1617-1682). Pour la rédaction des manuels de pratique, indispensables à la performance des rituels, la plupart d'entre eux ont été rédigés à l'instigation des autorités monastiques et, comme le précisent les colophons, résultent de multiples interventions auxquelles s'ajoutent souvent des remarques en petits caractères dues à de précédents utilisateurs.

Conscients de l'écrasante responsabilité qui était la leur pour assurer la transmission des traditions dont elles étaient dépositaires, les autorités religieuses nyingmapa se sont ingéniées à assurer la mise en place et le développement d'institutions au sein desquelles pouvait être assuré le cycle complet des études et c'est à ce besoin qu'a répondu l'ouverture de "collèges philosophiques » (bshad grwa) comme il en existe désormais à Shéchen.

À l'aube de ce XXI ${ }^{\mathrm{e}}$ siècle, à l'heure où se multiplient éditions, traductions, centres d'enseignements et où la diffusion par internet permet un accès direct à des sources autrefois réservées à un petit nombre, on peut se demander ce qui va advenir de ce rapport entre monachisme et érudition et si la langue tibétaine ne va pas désormais être cantonnée au seul usage liturgique.

Mireille HELFFER

Centre de recherche en ethnomusicologie - CNRS -

Paris Ouest Nanterre La Défense mic.helffer@magic.fr 


\section{Bibliographie}

N.B. Les références aux textes tibétains sont données au fur et à mesure du texte.

Blondeau Anne-Marie, 1976, "Les religions du Tibet ", in Encyclopédie de la Pléiade, Histoire des religions III, Paris, Gallimard, pp. 233-329.

Blondeau Anne-Marie, Schipper Kristofer, (éds.), 1988, Essais sur le Rituel vol. I-II. Colloque du centenaire de la section des Sciences religieuses de l'EPHE, Louvain-Paris, Bibliothèque de l'École des Hautes Études, coll. «Section des Sciences religieuses », XCII, XCV.

Cornu Philippe, 2001, Dictionnaire encyclopédique du bouddhisme, Paris, Éditions du Seuil.

-, 1997, Padmasambhava. La magie de l'Éveil, Paris, Éditions du Seuil, coll. "PointsSagesses».

Dudjom Rinpoche, Jikdrel Yeshe Dorje, 1991, The Nyingma School of Tibetan Buddhism. Its Fundamentals and History, (bDud 'joms 'jigs bral ye shes rdo rje), traduit et édité par Gyurme Dorje avec la collaboration de Matthew Kapstein, Boston, Wisdom Publications.

HeLFFer Mireille, 1994, Mchod-rol. Les instruments de la musique tibétaine, Paris, CNRSÉditions / Éditions de la MSH.

-, 1995, «Quand le terrain est un monastère tibétain », Terrains, 8, "Cahiers des musiques traditionnelles ", pp. 69-84.

-, 1998, «Du son au chant vocalisé : la terminologie tibétaine à travers les âges (VIII $\mathrm{xx}^{\mathrm{e}}$ siècle) ", Cahiers des musiques traditionnelles, Paroles de musiciens, 11, pp. 141162.

-, 2004a, " Traditions musicales dans un monastère du bouddhisme tibétain ", L'Homme, 171-172, pp. 173-196.

-, 2004b, "Regards sur les formes dans la musique rituelle du bouddhisme tibétain ", Cabiers des musiques traditionnelles, Formes musicales, 17, pp. 261-296.

-, 2004c, Musiques du toit du monde: l'univers sonore des populations de culture tibétaine, Paris, l'Harmattan, "Musique et Musicologie».

-, 2005, «Musique et bouddhisme : un rituel tibétain ", in Musiques : une encyclopédie pour le XXI ${ }^{e}$ siècle, Tome 3, Musiques et Cultures, Paris, Actes Sud-Cité de la Musique, pp. $420-438$.

HelfFer Mireille, Ricard Matthieu, 1997, CD Sounds of Tibet: Monastery of Shechen, Shechen Publications 001, Delhi \& Kathmandu [notice du CD conceived under the inspiration of Shechen Rabjam Rinpoche].

Kapstein Matthew, 2006, The Tibetans, Maiden, Oxford, Victoria, Blackwell Publishing.

Karmay Samten G., 1989, Le Manuscrit d'or : Visions secrètes du Ve Dalaï Lama, Paris, Éditions Findakly «coll. Patrimoines d'Orient».

KoHN Richard J., 2001, Lord of the Dancelthe mani rimdu Festival in Tibet and Nepal, New York, State University of New York Press, coll. «SUNY Series in Buddhist Studies ».

NeвеSкy-Wojкоwiтz René de, 1976, Tibetan Religious Dances, (Tibetan text and annotated translation of the 'chams yig, Christoph von Fürer-Haimendorf, ed., appendix by Walter Graf), The Hague, Mouton. 
I36 - ARCHIVES DE SCIENCES SOCIALES DES RELIGIONS

RICARD Matthieu, 1996, L'Esprit du Tibet: la vie et le monde de Dilgo Khyentsé maître spirituel, Paris, Seuil.

-, 1999, Moines danseurs du Tibet, Paris, Albin Michel.

Smith Gene E., 2001, Among Tibetan Texts. History and Literature of the Himalayan Plateau, (ed. Kurtis R. Schaeffer, foreword Jeffrey Hopkins), Boston, Wisdom Publications.

Snellgrove David, 1957, Buddhist Himalaya: Travels and Studies in Quest of the Origins and Nature of Tibetan Buddhism, Oxford, Bruno Cassirer.

-, 1987, Indo-Tibetan Buddhism. Indian Buddhists and their Tibetan Successors, London, Serindia Publications.

STEIN Rolf, 1957, « Le linga des danses masquées lamaïques et la théorie des âmes ", SinoTibetan Studies, V/3-4, pp. 200-234.

-, [1962] 1981, La civilisation tibétaine, Paris, Le Sycomore-L'Asiathèque.

TSEPAK Rigzin, Tibetan-English Dictionary of Buddhist Terminology, Dharamsala, Library of Tibetan Works and Archives.

En sus de nombreux glossaires figurant dans les traductions anglaises et françaises d'ouvrages tibétains.

\section{Résumé}

La rencontre de l'ethnomusicologue avec la filiale d'un monastère de l'est du Tibet, le monastère de Shéchen, implanté en exil à Bodnath (Népal), permet de rendre compte de l'engagement spirituel et cultuel inlassable d'un des plus grands maîtres tibétains du XXe siècle, Dilgo Khyentse Rinpoche (1910-1991). En dépit des épreuves traversées, ce maître a œuvré jusqu'à son dernier jour pour la propagation du bouddhisme en promouvant éditions de textes fondamentaux, constructions, accueil et formation des novices et futurs professeurs. Il a dispensé nombre d'enseignements et s'est attaché à favoriser la perpétuation des rituels par la publication des manuels de chant propres à la tradition nyingmapa. Son action se poursuit aujourd'hui par l'intermédiaire de son petit-fils Rabjam Rinpoche.

Mots-clés : Tibet, bouddhisme, musique, notations neumatiques, danses, transmission.

\section{Abstract}

The meeting between the ethno-musicologist with the joint community of the EasternTibetan monastery of Shechen, in exile, enables us to render a good account of the tireless spiritual and cultual commitment of Dilgo Khyentse Rimpoche (1910-1991), one of the greatest Tibetan masters of the $20^{\text {th }}$ century. Despite the ordeals he suffered, he worked till his last days to the propagation of Buddhism promoting the editions of classical texts, the reception and training of novices and future teachers. He gave many lessons and favoured the perpetuation of rituals by publishing manuals of the Nyingmapa tradition songs. His action is carried on today by his grandson Rabjam Rinpoche.

Key words: Tibet, Buddhism, music, neumatic notations, dances, transmission. 


\section{Resumen}

El encuentro del etnomusicólogo con la filial de un monasterio del este del Tíbet, el monasterio de Schenen, implantado en el exilio en Bodnat (Nepal), permite dar cuenta del compromiso espiritual y cultural incansable de uno de los más grandes maestros tibetanos del siglo XX, Dilgo Khyentse Rinpoche (1910-1991). A pesar de las pruebas que tuvo que atravesar, este maestro trabajó hasta su ultimo día para la difusión del budismo, promoviendo ediciones de los textos fundamentales, construcciones, acogida y formación de novicios y futuros profesores. Dispensó numerosas enseñanzas y se abocó a favorecer la preservación de los rituales para la publicación de los manuales de canto propios de la tradición nyingmapa. Su acción se continua hoy a través de su nieto Rabjam Rinpoche.

Palabras clave: Tíbet, budismo, música, anotaciones neumáticas, danzas, transmisión. 
